(c) 2021 by «D. Rogachev NMRCPHOl» Received 14.01.2021 Accepted 02.02.2021

Контактная информация: Mehdi Gholamzadeh Baeis, Department of Radiology, Imam Hossein Hospital, Shahid Beheshti University of Medical Sciences, Iran, Tehran E-mail: Dr.M.Gholamzadeh@Gmail.Com

\section{Case report of mesenteric lymphadenopathy in Gaucher disease: rare and benign complication}

\author{
M. Khalili' ${ }^{1}$, M. Gholamzadeh Baeis ${ }^{1}$, M. Alaei ${ }^{2}$ \\ ${ }^{1}$ Shahid Beheshti University of Medical Sciences, Iran, Tehran \\ 2Mofid Children's Hospital, Shahid Beheshti University of Medical Sciences, Iran, Tehran
}

\begin{abstract}
A 10-year-old boy known case of Gaucher disease about 2 years ago was admitted with nausea, vomiting and epigastric pain from 3 days before hospitalization to Mofid Children's hospital, Tehran, Iran. On the initial examination, no abnormalities were found except for splenomegaly and brief tenderness on the spleen. In secondary paraclinical evaluations (with Ultrasound and Ct-scan), we noticed mesenteric lymphadenopathy in the patient. The lesions remained unchanged and no significant changes were observed in the clinical and laboratory evaluations of the patient during one-year follow-up period. This is a rare and benign manifestation of Gaucher disease, which is usually monitored and does not require any intervention without signs of malignancy or severe space occupying effects
\end{abstract}

Key word: mesenteric lymphadenopathy, Gaucher disease, pediatric patient

Khalili M., et al. Pediatric Hematology/Oncology and Immunopathology. 2021; 20 (1): 168-169. DOI: $10.24287 / 1726-1708-2021-20-1-168-169$

\begin{abstract}
3 aucher disease is a metabolic disorder due to a deficiency of the $\beta$-glucocerebrosidase enzyme, which leads to the glucocerebroside accumulation in tissue macrophages of liver, spleen, and bone marrow, lungs, kidneys and intestines. In this disease, fat accumulates in the lysosomes of macrophages and is not metabolized [1]. The main features of the disease are anemia, bone lesions, neurological disorders and hepatosplenomegaly. Moreover the disease is associated with rare complications, such as mesenteric lymphadenopathy, which only 26 cases have been reported worldwide [2]. In the present study we describes a pediatric patient with Gaucher disease, who had mesenteric lymphadenopathy as a rare and benign manifestation of Gaucher disease.
\end{abstract}

\section{CASE REPORT}

A 10-year-old boy known case of type1 Gaucher disease (homozygous c.1448T>C (p.L483P); Lyso-Gl-1 - $875.5 \mathrm{ng} / \mathrm{ml}$ (cut of value $0-4.0 \mathrm{ng} / \mathrm{ml}$ )) about 2 years ago has been under Enzyme Replacement Therapy (ERT). The treatment was received incompletely. He was admitted with nausea, vomiting and epigastric pain 3 days before hospitalization, to the Mofid Children's Hospital, Tehran, Iran. On the initial examination, no abnormalities were found except for splenomegaly and brief tenderness on the spleen. To complete the initial assessment for the patient, laboratory tests and ultrasound of the abdomen were performed, the findings included: WBC 5,7 $\times 10^{9} / \mathrm{L}$ (Neut 55\%, Lym 37\%), PLT $138 \times 10 \% / \mathrm{L}$, Hb $114 \mathrm{~g} / \mathrm{L}$, liver function test and amylase were in normal limit.
Evidence of lobulated large mesenteric mass measuring about $91 \times 57 \mathrm{~mm}$ by ultrasound was depicted (figure 1).

For better assessment of the lesion, CT-scan of the abdomen and pelvis with IV contrast was requested for the patient, which showed the following findings.

Congluromated lymphadenopathy containing coarse calcification and area of cystic degeneration was detected which encased SMA (figure 2). By given the history, clinical condition, laboratory data (normal CBC, ESR and LDH) and imaging, mesenteric lymphadenopathy as a rare and benign manifestation in Gaucher disease was considered. Due to the strong clinical suspicion of the benign process and the high risk of surgery for the patient, sampling and surgery were not performed. It is noteworthy that taking an

Figure 1

In Ultrasound, evidence of lobulated large mesenteric mass measuring about $91 \times 57 \mathrm{~mm}$ in the epigastric region by spreading to the umbilical region was seen

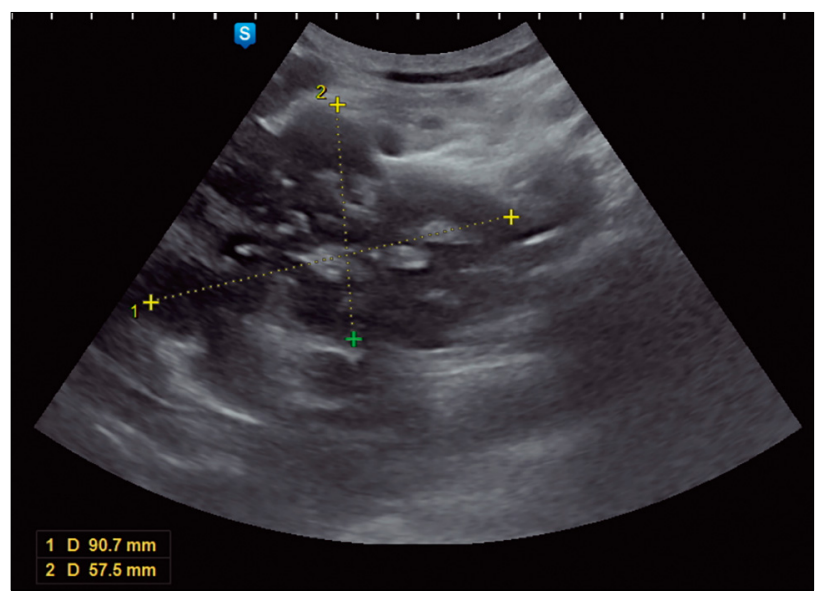


antacid after one week relieved the patient's epigastric pain. The patient was also followed up by serial ultrasounds over the course of a year, and the lesions remained unchanged and no significant changes were observed in the patient's clinical and laboratory evaluation during this one-year follow-up period.

\section{DISCUSSION}

Accumulation of glycosphingolipids in the mononuclear phagocyte system, may progress to disabling and potentially life-threatening complications when left undiagnosed and untreated(1). Mesenteric lymphadenopathy is a rare and benign manifestation in pediatric patients with Gaucher disease, which is generally refractory to ERT and substrate reduction therapies [3].

The clinical implication of this condition is undetermined, with no consensus on treatment strategies. However, this condition can reflect the progression of Gaucher disease. Moreover, it can be accompanied by the serious complication, protein-losing enteropathy [4].

Figure 2

Conguloromated lymphadenopathy containing coarse calcification and area of cystic degeneration was detected which encased SMA
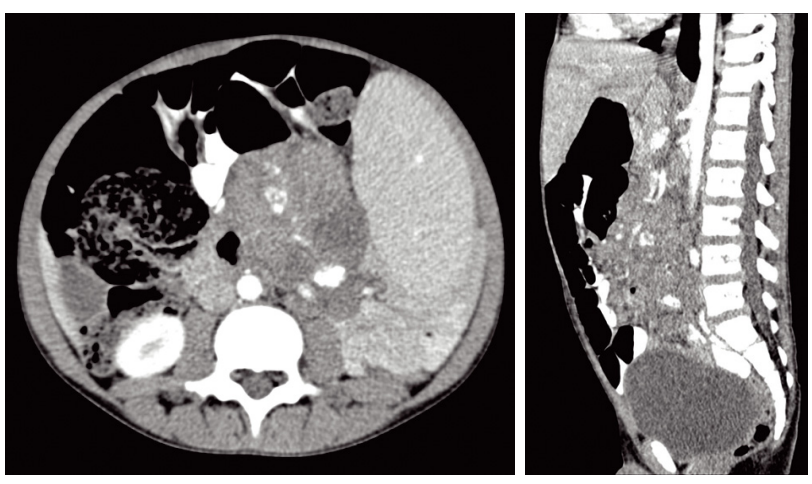

Despite successful treatment with ERT, several cases of this manifestation have been reported. It is not clear why ERT is not effective in these rare cases. But it may be because the lymph nodes do not have access to this type of treatment [3].

In a few studies biopsy of lymph nodes was performed which revealed no malignancy [4-6].

The main differential diagnosis is lymphoma which is still a rare manifestation in Gaucher disease, however calcification in lymphomatous mass is only seen after therapy [7].

Thus, according to the results of our study and other similar studies, misdiagnosis as a malignant tumor and incorrect treatment can be avoided with increased recognition and knowing better about this rare manifestation. Surgical interventions may only be required if malignant lesions are highly suspected and mass effects are present on other organs due to the size of the lymph nodes. Further reporting and evaluation, and longer follow-up of similar cases, are needed to obtain more accurate information.

\section{CONCLUSION}

Our study showed that mesenteric lymphadenopathy can be a benign and rare manifestation in Gaucher disease and does not require immediate intervention. It can be monitored and if it leads to more complications, then appropriate interventions could be considered.

\section{FUNDING}

Not specified.

\section{CONFLICT OF INTEREST}

The authors declare that there is no conflict of interest.

ORCID

Gholamzadeh Baeis M. ORCID: http://orcid.org/0000-0002-1696-6307

\section{References}

1. Carubbi F., Cappellini M.D., Fargion S., Fracanzani A.L., Nascimbeni F. Liver involvement in Gaucher disease: A practical review for the hepatologist and the gastroenterologist. Dig Liver Dis 2020; 52 (4): 368-73.

2. Ye Z.-X., Gao X., Qu Q., Ye X., He X.-D. Gaucher Disease with Mesenteric Lymphadenopathy: A Case with 13-year Follow-up. Chin Med J 2016; 129 (20): 2502-3.

3. Simpson E.A., Jaring M.R., Andronikou S. Massive Mesenteric Lymphadenopa- thy Causing Protein-losing Enteropathy in Gaucher Disease. J Pediatr Hematol Oncol 2017; 39 (5): e300-1.

4. Lee B.H., Kim D.Y., Kim G.H., Cho K.J., Yoon H.K., Yoo H.W. Progressive mesenteric lymphadenopathy with protein-losing enteropathy; a devastating complication in Gaucher disease. Mol Genet Metab 2012; 105 (3): 522-4.

5. Abdelwahab M., SeifEldien H.M. Mesenteric and Mediastinal Lymphadenopathy in Egyptian Children With Gaucher Disease Types 1 and 3 Treated With
Enzyme Replacement Therapy. J Pediatr Hematol Oncol 2015; 37 (5): e31622.

6. Burrow T.A., Cohen M.B., Bokulic R., Deutsch G., Choudhary A., Falcone R.A. $J$, et al. Gaucher disease: progressive mesenteric and mediastinal lymphadenopathy despite enzyme therapy. J Pediatr 2007; 150 (2): 202-6.

7. Frampas E. Lymphomas: Basic points that radiologists should know. Diagnn Interv Imaging 2013; 94 (2): 131-44. 